\title{
Ausgewählte linguistische Entwicklungen in der Erzählforschung
}

Die linguistische Erzählforschung wendet ihre Aufmerksamkeit drei Kernbereichen zu, die auch als Entwicklungsphasen mit eigenen Interessensschwerpunkten verstanden werden können: a) die schriftliche Erzähltextanalyse unter dem Einfluss des Strukturalismus und zu Teilen der Literaturwissenschaften, b) die mündliche Erzählforschung unter dem Einfluss der pragmatischen Wende und der Gesprächsanalyse c) sowie das Erzählen in den Neuen Medien bzw. digitalen Medien insbesondere unter dem Einfluss der Computer-vermitteltenKommunikation (computer-mediated-communication (CMC)). Durch die spezifischen Interessensschwerpunkte entstanden unterschiedliche Gewichtungen der Perspektiven und Beschreibungsansätze, die zwar zu verschiedenen Forschungsrichtungen mit ihren eigenen Untersuchungsmethoden führen, sich aber durchaus auch gegenseitig ergänzen können.

Da ich die Daten dem Internet entnommen habe, sind sowohl Errungenschaften der mündlichen Erzählforschung (Film und Bewegtbild), als auch diejenigen zu den digitalen Medien für diese Arbeit unabdingbar. Im Zusammenhang mit der mündlichen Erzählforschung gehe ich auf narrative ,Jobs‘ aus dem Modell zur Beschreibung von Erzählinteraktion von Hausendorf/Quasthoff (1996) ein. Da das Modell sich auf drei einflussreiche Strömungen der mündlichen Forschung stützt, erläutere ich zuvor deren primäres Forschungsinteresse (Abschn. 4.1). Im Anschluss daran nehme ich die Entwicklung der Computer-vermitteltenKommunikation, als eine Entwicklung der Erzählforschung in den Neuen Medien, in den Blick (Abschn. 4.2). In diesem Zusammenhang ist die Betrachtung von Erzählen als Kontinuum (Ochs/Capps 2001) in Verbindung mit dem Konzept der Small Story (Bamberg/Georgakopoulou 2008) von Interesse. 


\subsection{Mündliche Erzählforschung: narrative ,Jobs'}

In der Linguistik leitete die pragmatische Wende in den 1970er-Jahren einen Paradigmenwechsel ein, der von einer systemorientierten Sprachbetrachtung (Fix 2008a: 15) ,zu einer betont kommunikativ und funktional orientierten Linguistik“ (Heinemann/Viehweger 1991: 22) überwechselt. Seitdem betrachtet man in der Linguistik Texte nicht nur aus einer rein textinternen Perspektive, sondern bezieht auch Textexterna (z. B. Situation und Kontext) ein ${ }^{1}$. Zusätzlich rückt der Handlungscharakter (informieren, beschreiben, darstellen, überzeugen u. a.) von Schriftlichem und Gesprochenem in den Mittelpunkt des Interesses. Ausgehend von dem Bewusstsein dafür, dass Erzählen eine Alltagspraktik ist (Spieß/Tophinke 2018: 193; Schneider 2017: 121), entwickelte sich eine Neuorientierung, die insbesondere mündliche Erzählungen als Forschungsbereich der Linguistik etablierte. Grundsätzlich kann das Interesse der mündlichen Erzählforschung sowohl textorientiert als auch dialogorientiert sein (vgl. Kotthoff 2017: 3; Becker/Stude 2017: 1; Schneider 2017: 116). Methodologisch sieht Quasthoff (2001: 1296) die mündliche Erzählforschung in hohem Maß von der Konversationsanalyse, der Ethnographie des Sprechens und der linguistischen Diskursanalyse beeinflusst. Durch das Wirken dieser Strömungen nahm die Erzählforschung eine neue Richtung ein. Ihr Einfluss wird im Folgenden kurz benannt, da Hausendorf und Quasthoff (1996) darauf ihr Modell zur Beschreibung von Erzählinteraktion stützen, auf dessen Parameter ,Dramatisieren' und ,Thematisieren 'ich mich in den empirischen Kapiteln beziehe ${ }^{2}$.

Der Konversationsanalyse spricht Quasthoff (2001) den umfangreichsten Einfluss auf die mündliche Erzählforschung zu. Ihr Einfluss beruht - so Quasthoff auf der Entdeckung des prozesshaften und sequenziellen Charakters jeglicher Interaktion sowie der Konzeptualisierung des Erzählens als dialogischem Prozess.

\footnotetext{
${ }^{1}$ In der Textlinguistik zeigten sich erste methodische Ansätze zum Umgang mit dem neuen Textverständnis bereits Mitte der siebziger Jahre bei Utz Maas (1972: 189 ff.), der den Einbezug der sozialen Situation in die Textbeschreibung forderte oder bei Ehlich und Rehbein (1972: 208 ff.) sowie bei Hartung (1974: 828), die die soziale Interaktion in Form des Kommunikationsereignisses in den Blick nahmen. Der erste funktionale Gesamtansatz stammt von Brinker (1985), der kontextuelle Merkmale und die Textfunktion als grundlegende Analyseschritte in sein Konzept integrierte. (Heinemann 2008: 139)

${ }^{2}$ Z. B. in Kap. 8, indem ,Dramatisieren“ als ein Aspekt der Beschreibungsdimensionen zur narrativen Stilisierung angewandt wird, oder Ergebnisse zum Thematisieren in verschieden narrativen Vermittlungsvorkommen (Kap. 9).
} 
Unter dieser Perspektive erlangten das Redemanagement und die Zuhöreraktivität an Bedeutung ${ }^{3}$. In diesem Fall bezieht sich die Forschungserkenntnis auf die Organisation der Beteiligungsrollen im Gespräch oder die „Zusammenarbeit" zwischen Erzähler/innen und Hörer/innen (z. B. Mandelbaum 1993; Duranti 1986; Quasthoff 1981). Das verdeutlicht, dass sich nicht nur die Erzählerin oder der Erzähler, sondern auch die Zuhörer/innen aktiv an der Erzählung beteiligen, z. B. in Form gemeinsamer Aufarbeitung, Würdigung und dem Abschluss der Erzählung (Sacks 1995) ${ }^{4}$. Dabei ist die Konversationsanalyse nicht speziell am Erzählen selbst, „,sondern an talk-in-interaction“ (Quasthoff 2001: 1298, Hervorhebung i.O.) interessiert, worin Erzählungen einen Untersuchungsbereich von vielen darstellen. Die Untersuchungsfelder des mündlichen Erzählens unter der konversationsanalytischen Perspektive sind breit gefächert. Sie befassen sich neben Alltagserzählungen ebenso mit dem Erzählen in Institutionen wie z. B. der Arzt-Patienten-Interaktion (Bliesener 1980; Gülich 2010), dem Therapiegespräch (Boothe 2004; Flader/Giesecke 1980), dem Erzählerwerb von Kindern, der Unterrichtskommunikation (Ehlich 1983a; Boueke et al 1995; Ohlhus 2014) oder der Interaktion in der Elternsprechstunde (Kotthoff 2015).

Den Einfluss der Diskursanalyse auf die mündliche Erzählforschung sieht Quasthoff in der „Herausarbeitung der geordneten Strukturiertheit auch übersatzmäßiger Einheiten“ (ebd. 2001: 1296). Das unterstreicht beispielsweise die Veröffentlichung Erzählen in digitalen Diskursen des Instituts für Deutsche Sprache (OPAL). In diesem Sinne leiten die Autorinnen den Band folgendermaßen ein:

„Ein Ziel, welches viele Spielarten der Diskurslinguistik teilen, ist die Rekonstruktion von Denk- und Vorstellungswelten bzw. Wissensbeständen auf transtextueller Ebene. Daher wollen wir in diesem Band das transtextuelle Muster des Narrativs vorstellen und seine Anwendbarkeit für digitale Diskurse an Fallbeispielen demonstrieren.“ (Mell/Gredel 2018: 3)

Die Ethnografie ist schwerpunktmäßig an kulturvergleichenden Fragestellungen und weniger an Erzählungen interessiert. Dennoch hat sie auf die mündliche Erzählforschung durch die Kontextualisierungsanalyse Einfluss genommen. Im Gegensatz zu der Diskursanalyse, die überwiegend an der globalen Strukturierung

\footnotetext{
${ }^{3}$ Siehe hierzu bspw. Arbeiten von Rehbein 1980; Quasthoff 1980; Hausendorf/Quasthoff 1996; Schegloff 1997.

${ }^{4}$ Die Konversationsanalytiker der „ersten Stunde“ (Kotthoff 2017: 5) waren Jefferson (1978), Sacks (1974) und Schegloff (1980).
} 
von Erzählungen interessiert ist, fokussieren Forschungsbeiträge über das Phänomen der Kontextualisierung lokale Strukturen, wie beispielsweise prosodisch markierte Formen der Redewiedergabe. Mit dieser Technik können Erzähler/innen die Redewiedergabe mittels sprachlicher Mittel ihrer/ihren ursprünglichen Verfasser/innen zuordnen, z. B. durch Varietätenwechsel (z. B. Standardsprache vs. Dialekt, Keim 1993) oder prosodisch expressive Merkmale, die die Stimme des/der Sprecher/in imitieren (Günthner 2007). Auf der Basis von Erkenntnissen aus den drei benannten Forschungsrichtungen entwickeln Hausendorf/Quasthoff (1996) ein Modell zur Beschreibung von Erzählinteraktion, aus dem ich in der empirischen Analyse die Parameter ,Dramatisieren“ und ,Thematisieren einbeziehe.

Hausendorf/Quasthoff (1996) entwickeln ihr Modell im Rahmen einer 15jährigen, empirisch ausgerichteten Forschungsarbeit über Sprachentwicklung und Interaktion. Die Rahmenbedingungen für das Modell stellen die Merkmale aus den drei gerade beschriebenen Forschungsrichtungen dar: Prozessualität/Sequenzialität, Interaktivität und Kontextualität. Grob umrissen bedeutet das, dass min. zwei Teilnehmer/innen die narrative Struktur gemeinsam turn-by-turn aufbauen. Daraus entwickelt sich eine sequenziell und prozessual repräsentierte narrative Struktur, die ,immer adressatenbezogen kontexualisiert [ist]“ (Quasthoff 2001: 1301). Aufgrund der gemeinsamen Beteiligung an der narrativen Struktur, wechseln Sprecher/innen und Zuhörer/innen ihre Rollen. Das stellt wiederum spezielle Anforderungen an die Organisation des Sprecherwechsels. In diesem Prozess manifestieren sich narrative Strukturen an der Oberfläche des Interaktionsgeschehens. Ausgehend davon erarbeiten Hausendorf/Quasthoff anhand empirischer Daten das Modell zur Beschreibung von Erzählinteraktion. Zwar ist das Modell auf die mündliche Erzählsituation ausgerichtet, doch haben sich Gülich/Hausendorf (2001) die Frage gestellt, ob man die Ebene der Hörer/Sprecherinteraktion nicht ebenfalls für die Kommunikation schriftlicher Texte fruchtbar machen kann. Ihre Schlussfolgerung lautet:

\footnotetext{
„Geht man davon aus, dass die narrationsspezifischen Aufgaben in und mit Kommunikation bearbeitet und gelöst werden, muss und kann man diese Aufgaben anhand der sprachlichen Erscheinungsformen dieser Kommunikation, also anhand der Texte im engeren Sinne, rekonstruieren und vice versa konkrete Textelemente und -strukturen als Formen und Mittel ihrer Bearbeitung und Lösung nachweisen.“ (Gülich/Hausendorf 2000: 375, Hervorhebung U.A.)
}

Der Argumentation zufolge ist somit die Textoberfläche jener Bereich, an dem sich die Angemessenheit der Aufgabenstellung ,Erzählung' bewähren muss. Des Weiteren setzt eine interaktive Modellkonzeption voraus, dass nicht nur 
der/die Erzähler/in, sondern auch ihre Zuhörer/innen den Redegegenstand als Erzählung erkennen. Das bedeutet, dass charakteristische Merkmale von Erzählungen in Form narrativer Aufgaben dem Gegenstand entsprechen und somit auch für schriftliche Erzählungen konstitutiv sein müssen (Gülich/Hausendorf 2000: 375 f.). Das Modell zur Beschreibung der Erzählerinteraktion beinhaltet sogenannte ,Jobs'. Darunter verstehen Quasthoff und Hausendorf bestimmte strukturelle Aufgaben innerhalb der narrativen Interaktion. Ihre Abfolge ,hat den Status einer allgemein interaktiv hochgradig erwartbaren strukturellen Regularität" (Hausendorf/Quasthoff 1996: 127). Deutlich wird das daran, dass es für gewöhnlich einer Begründung bedarf, wenn die Abfolge verändert wird. Insgesamt beinhaltet das Modell fünf globalstrukturelle Elemente in linearer Abfolge: a) Darstellen von Inhalts- und/oder Formrelevanz, b) Thematisieren, c) Elaborieren / Dramatisieren, d) Abschließen, e) Überleiten. Da das Modell an der Interaktion ausgerichtet ist, nehme ich in der folgenden Ausführung allgemeingültige Aspekte auf, die sowohl für das Mündliche, als auch das Schriftliche gelten (vgl. Gülich/Hausendorf 2000: 376).

Das Herstellen einer Inhalts- und/oder Formrelevanz bezieht sich darauf, dass eine Erzählung irgendwie angebahnt werden muss. Das bedeutet, der/die Erzähler/in etabliert einen Rahmen, innerhalb dessen er/sie auf die Erledigung der narrativen Thematisierung zurückkommt. In den erhobenen Daten betrifft das einen inhaltlichen Bezugsrahmen, der den Erzähler/innen dazu dient, das betreffende Ereignis später vorzustellen und weiter auszuarbeiten. Die Funktion der narrativen Aufgabe ist es, die Rezipient/innen darauf vorzubereiten, dass ein ,großes Paket` folgt. Das Thematisieren erfolgt durch die Eröffnung der Erzählung. Es geht darum, die narrative Diskurseinheit selbst konditionell relevant zu machen, worüber der Zugzwang entsteht, eine Erzählung anzuschließen (Hausendorf/ Quasthoff 1996: 130). In den Daten scheinen diese zwei ersten Elemente des Modells zusammenzufallen. So ist bereits die Überschrift oder der Aufmacher eine Form des Thematisierens, was anhand von Gliederungssignalen in Form grafischer Mittel (Schriftgröße, -farbe, Fettdruck, Zeilenumbrüche) deutlich wird. Ihre Funktion liegt gerade darin, die Aufmerksamkeit der Rezipient/innen zu wecken und bei ihnen um einen Zeitvorschuss für den Folgetext $\mathrm{zu}$ werben (vgl. Hausendorf/Gülich 2000: 378). Andererseits legen die Erzähler/innen auf diese Weise den inhaltlichen Rahmen fest, in dem sich die folgende Erzählung entwickelt. Elaborieren und Dramatisieren sind genau genommen erzählspezifische Aufgaben im Sinn der Kernaufgabe des Erzählens (Hausendorf/Quasthoff 1996: 130). Der ,Job“ bedeutet, dass ein Erzähler das entsprechende Ereignis „in einer übersatzmäßigen Einheit narrativ entfaltet“ (ebd.). Das Elaborieren bedeutet, 
eine situativ angemessene und adressatenbezogene Rekonstruktion der Ereignisse. Das Dramatisieren bezieht sich auf die Relevanz, die für gewöhnlich in dem vermittelten Erzählenswert liegt (Quasthoff 2001: 1303). Für die Daten wurden diesbezüglich anhand des Verortungsschemas narrative Indikatoren bestimmt. Auf der inhaltlichen Ebene signalisieren die Merkmale das Erzählenswerte, das in der Erzähleröffnung irgendwie greifbar gemacht und innerhalb der Dramatisierung nun Schritt für Schritt eingelöst werden muss. Charakteristische Mittel des Dramatisierens in schriftlichen Erzählungen sind das historische Präsens, die direkte Rede und die Lexikalisierung der Wahrnehmungs- und Erlebnisperspektive (Hausendorf/Gülich 2000: 379). Das Abschließen ist der „Übergang von der narrativen Diskurseinheit zum anschließenden Turn-by-turn-talk“ (Hausendorf/Quasthoff 1996: 380). Es wird bereits innerhalb der Erzählung mit semantischen und/oder strukturellen Mitteln kenntlich gemacht oder kann - wie in einigen Daten - beispielsweise mithilfe einer Abschiedsformel erfolgen, wie sie in einem Brief erwartet wird: Bis bald eure [...]. Inhaltlich kann der Abschluss schon im Rahmen einer Ereignisbeschreibung signalisiert werden, z. B. eine Veranstaltung die zu Ende geht. Das Überleiten gewährleistet den Anschluss an den nun wieder einsetzenden turn-by-turn-talk und somit die Aufhebung der interaktiven, narrativen „Sonderregeln“ (Hausendorf/Quasthoff 1996: 132). Dieser „Job“ ist allerdings für die vorliegenden schriftlichen Erzählungen weniger relevant. Möglicherweis könnte man hierunter abschließende Verlinkungen auf weitere Unternehmensseiten, z. B. die Karriererubrik, U.-Homepage u. a. verstehen.

\subsection{Erzählforschung zu den digitalen Medien}

Burger/Luginbühl (2014: 445) schlagen zusammen mit Holly/Jäger (2011) vor, anstatt von Neuen Medien alternativ von digitalen Medien zu sprechen, da jede Epoche neue Medien hervorbringe (z. B. in den 1980er-Jahren der Videorekorder). Entsprechend gilt je nach Betrachtungszeitpunkt ein Medium heute nicht mehr als ,neu“. Ich bezieht mich in meiner Arbeit auf das Medium ,Internet“, das sich dadurch auszeichnet, dass es Text, Bild, Ton, Grafiken u. a. kombinieren sowie Daten digital speichern und mittels Übertragungsnetze verbreiten kann. Da das Integrieren von Bildern oder Grafiken bereits in den Printmedien möglich war/ist, liegt das Neue vor allem darin, dynamische Elemente wie „Animation, Film sowie sprachliche[] und nicht-sprachliche[] akustische Zeichen" (Adamzik 2016: 62) einzubinden.

Mit dem Erzählen in digitalen Medien befasst sich die in den 1990er-Jahren aufgekommende Digitale Narratologie. Sie dokumentierte und theoretisierte die 
damals neuen künstlerischen Formen in ihrem erzählerischen Potential innerhalb digitaler Medien. Hierfür nahm sie zu Beginn insbesondere fiktionale Erzählungen in den Blick. Das führte allerdings dazu, dass sie zeitgenössische Gattungen (bspw. persönliche Blogerzählungen) als Forschungsgegenstand weitgehend exkludierte. Zeitgleich wurde die Computer-vermittelte-Kommunikation (computer-mediated-communication $(C M C)$ ) ein interdisziplinäres Forschungsfeld (Linguistik, Medienwissenschaften, Soziologie, Psychologie). Die Forscher/innen richteten ihre Aufmerksamkeit in dieser „ersten Welle“ (Androutsopoulos 2006: 420) darauf, generische Muster (bspw. die Übertragung von turn-taking Mustern der face-to-face Kommunikation) in verschiedenen Genres aufzudecken und zu erforschen, wie sich Normen für die Online-Interaktion etablieren. (Page 2013: 3 f.) Diese Betonung medienspezifischer Merkmale spiegelt sich auch - so Androutsopoulos (2006: 420) - in Bezeichnungen wie electronic language und computer-mediated register. In der ,Zweiten Welle“ verlagerte sich dann die Forschung auf das Zusammenspiel von technologischen, sozialen und kontextuellen Faktoren (Androutsopoulos 2006: 421), also mehr in die Richtung von benutzerzentrierten Darstellungen der CMC. Untersuchungen von Erzählungen innerhalb der beiden Phasen wie z. B. Krankheitsnarrative in Diskussionsforen (McLellan 1997) oder Small Stories in E-Mails (Georgakopoulou 2004, 2007; Heyd 2008) waren jedoch überwiegend darauf ausgerichtet, die Daten innerhalb des digitalen Kontextes zu erfassen und weniger darauf ausgerichtet, wie narrative Genres in diskursiven Online-Kontexten gestaltet werden. (Page 2013: 5) Entsprechend konzentrierten sich Studien zum digitalen Erzählen kaum darauf, wie diese Geschichten tatsächlich erzählt werden und welche textuellen und interaktionellen Aspekte sie ausmachen (Georgakopoulou 2013: 701).

In den letzten Jahren rücken Untersuchungen zu diesen Fragestellungen vor allem in Bezug auf die sozialen Medien in den Mittelpunkt. Die Bandbreite der Forschung ist umfangreich. Sie reicht - um nur einige Beispiele zu nennen - über die Analyse von Web-Serien, Games (Domsch 2012), Viral Spots (vgl. Eick 2014), YouTube (Rymes 2018, Beißwenger [2010] 2019), Facebook (Tagg/Seargant 2016; Androutsopoulos 2014b, 2013; Brook/Locher 2010) und Twitter (Klemm 2018; Weidacher 2018; Dayter 2015; Klein 2013; Page 2013) bis zu Erzählungen in Internetforen und Weblogs (Klemm 2018; Puschmann 2009, 2010, 2012; Rinke 2012; Tophinke 2009, 2017). So untersucht beispielsweise Puschmann (2010) Unternehmensblogs (corporate blog) von US-amerikanischen Unternehmen. Er richtet sein Interesse u. a. auf kommunikative Funktionen in Blogs von einzelnen und institutionellen Nutzer/innen (ebd. 2010: 123). Seine 
Untersuchung mündet in die Unterscheidung von Ego blogging und Topic blogging. Ersteres zeichnet sich durch die Wiedergabe von Erfahrungen, Gedanken und Gefühlen der Blogger aus, während zweites von der Arbeit, Unterhaltung, Geschäftlichem u. a. handelt. Damit sind wiederum verschiedene Funktionen (kontrollieren und dokumentieren eigener Denkprozesse vs. Beeinflussung anderer), Perspektiven (intern vs. extern) und auch sprachliche Merkmale verbunden. Dabei ordnet der Autor dem Ego blogging das Erzählen und den erzähltypischen „stream of consciousness“ (ebd.: 42) zu. Im Gegensatz dazu ist das Argumentieren der Kommunikationsmodus des Topic blogging. Tophinke (2009) setzt sich im Zusammenhang mit Wirklichkeitserzählungen (Klein/Martinez 2009) mit privaten Weblogs auseinander. Dabei spielt die grafische Textgestaltung eine besondere Rolle. Der Text wird durch das Textlayout und Bildelemente zu einem visuellen Objekt (angelehnt an die Betrachtung als "Schriftbildschirm“ nach Schmitz (2006)). Sie fungieren als Kontextualisierungsmittel und tragen zur Sinnkonstitution bei, z. B. indem durch (jugend-)kulturelle Ästhetik und Symbolik auf sozio-kulturelle Zugehörigkeit verwiesen wird. (Tophinke 2009: 253 f.) Linguistische Untersuchungen zu Facebook behandeln beispielsweise Themen wie die ,vernetzte Mehrsprachigkeit“" (Androutsopoulos 2013), gemeinsame Sprachpraktiken und sprachliche Repertoires innerhalb der sozialen Netzwerke (Androutsopoulos 2014a) oder Sprachhandlungen zur Identitätsbildung privater Facebook-Nutzer/innen (Tagg/Seargeant 2016; Brook/Locher 2010). Konkreten Praktiken des Erzählens auf Facebook widmet sich Page (2012) in einer Langzeitstudie. In dieser untersucht sie den Stil verfasster Erzählungen von Privatpersonen in Facebook-Updates (erste Erhebung 2008 von 1.000 Updates und die zweite Erhebung 2010 von 1.000 Updates). Sie kommt dabei u. a. zu dem Ergebnis, dass prinzipiell die Abwesenheit der face-to-face-Kommunikation für die Verfasser/innen das Risiko eines Gesichtsverlustes birgt. Das Risiko reduzieren diese dadurch, dass sie Alltägliches (also Unverfängliches) thematisieren, das jedoch auf den ersten Blick keine hohe Erzählwürdigkeit aufweist. Um die Erzählwürdigkeit dieser Erzählungen zu steigern und die Rezipierenden zu involvieren, treten in hohem Maße affektive und expressive Versprachlichungsstrategien auf (sprachliche Bewertungen, nonverbale Merkmale, Intensivierer und Verstärker). Auf diese Weise positionieren sie die „unscheinbaren“ Erzählanlässe für das Publikum als erzählenswert. Neben Facebook widmet sich Page (2015) ebenfalls dem Erzählen auf Twitter. Mit Blick auf klassisch narrative Differenzierungsmerkmale hält sie im Zuge ihrer Forschung über Social Media Storytelling fest, dass die Nutzer/innen verschiedener Internetplattformen (Facebook, Twitter u. a.) aufgrund der Aktualisierungsgewohnheiten weniger retrospektiv im Präteritum, sondern vielmehr im Präsens direkt aus ihrer Gegenwart heraus erzählen (ebd. 
2015: 335). Page nennt das daraus entstehende Muster im Zusammenhang mit privaten Posts ,real-time reporting of narrative events“ (Page 2013: 107). An dieser Stelle ist auf einen grundlegenden inhaltlichen Unterschied zwischen TwitterErzählungen von Privatpersonen und von Unternehmen in den erhobenen uRE hinzuweisen: In Erzählungen privater Nutzer/innen ist für gewöhnlich der „Fokus auf das empfindende Selbst“ (Puschmann/Heyd 2012: 188) gerichtet. Das ist in den erhobenen Daten von Unternehmen nicht in dem gleichen Ausmaße der Fall. Der Fokus eines empfindenden Selbst verlagert sich mit wenigen Ausnahmen weg von dem persönlichen Erleben der Erzähler/in hin zu der Vermittlung des Augenblicks für die Rezipient/innen. Die Forschungsergebnisse zu Twitter über Privatpersonen sind ein wichtiger Ansatzpunkt für meine Untersuchung, allerdings müssen sie immer wieder vor dem institutionellen Hintergrund betrachtet werden. Phänomene des Erzählens in den sozialen Medien, vornehmlich auf Facebook und Twitter, haben in Bezug auf „Personen“ (also nicht von Institutionen wie Unternehmen) neben Page ebenfalls Georgakopoulou $(2004,2015)$ unter der Bezeichnung breaking news, Dayter (2015: 21) als Delayed Resolution Narrative (siehe auch zu Twitter und Small Story Dayter 2014) oder auch Puschmann/Heyd (2012) beschrieben. Die Autor/innen stellen in Übereinstimmung mit den von mir erhobenen Daten fest, dass bezeichnenderweise bruchstückhafte Erzählungen auf Twitter bestehen (Puschmann/Heyd 2012: 175, 180; Georgakopoulou 2014: 11; Page 2015: 330, 345; Dayter 2015: 19). In der Erzählforschung wird das bislang vor allem für mündliche Gespräche in der face-to-face Kommunikation untersucht ${ }^{5}$. Auch wenn in den letzten Jahren das Forschungsdesiderat zunehmend mehr in den Blick rückt, werden überwiegend Erzählfragmente von Privatpersonen in den sozialen Medien untersucht. Der Anwendungsbezug zu Erzählungen von Unternehmen in den sozialen Medien, die nicht explizit der Produktwerbung zuzuordnen sind, ist derzeit in der Linguistik überschaubar.

Da digitale Technologien die Realisierungsoptionen von Erzähltexten erweitern und/oder verändern, steht die Erzählforschung vor Fragen wie: Worin unterscheiden sich Erzählungen in den digitalen Medien von anderen? Wie werden Erzählungen in den digitalen Medien umgesetzt? Wie beeinflussen sie das Erzählen? Welche semiotischen Modalitäten werden für was, wann verwendet? Einen kreativen Weg geht Lischeid (2018), um zu untersuchen, wo Inferenzen und Konvergenzen von Digitalität und Narrativität liegen. Er dreht in seinem

\footnotetext{
${ }^{5}$ Bspw. Gülich/Hausendorf (2012) „Träume im Gespräch: Linguistische Überlegungen zur Erzählbarkeit von Träumen“, Birkner (2013) „Erzählfragmente: narrative Funktionalisierungen zur Lösung der schweren Beschreibbarkeit von Schmerzempfindungen“, Kotthoff (2018) „Nicht ausgebaute Erzählungen“ im institutionellen Kontext (Schule und Arzt-PatientenGespräch).
} 
Artikel Digitales Erzählen - im Spiegel analoger Gegenwartsliteratur sozusagen den Spieß um. Hierfür untersucht er Romane und Narrative der Gegenwartsliteratur (im Bereich von Popular-, Midcult- und Hoch-Literatur (ebd. 2018: 9) vor der Fragestellung, inwiefern sie die printmedialen Oberflächen (Layout, SchriftBild-Kombinationen, Typografische Mittel, Darstellungsperspektiven u. a.) von „Bildschirm-“ bzw. „Monitor-Erzählen“ aus digitalen Umgebungen simulieren. Lischeid kommt zu dem Schluss, dass die Verfahren teils gegeneinander ausgespielt werden und teils miteinander verschmelzen. Sie sind dem Autor zufolge „,in der logischen Figur einer sich wechselseitig überkreuzenden Verweisung angelegt“" (Lidcheid 2018: 16). Daraus schließt er,

„dass jede wahre Analogizität nicht ohne Digitalität zu haben sei, so wie sich eben umgekehrt jede wahre Digitalität mit geprägt zeigen müsse durch ihren Anteil an anthropogener Kreativität, Originalität und Innovativität, Authentizität und Individualität [...].“(ebd.)

Diese Schlussfolgerung verdeutlicht den nicht zu vernachlässigenden Einfluss des analogen Erzählens auf das digitale, insofern als das Internet, bevor es ,völlig neue, eigenständige Formate entwickelte, [...] zunächst einmal alle bestehenden Formate einfach nur abgebildet [hat]" (Eick 2014: 26). Dadurch bestehen sowohl Gemeinsamkeiten und Ähnlichkeiten zu printmedialen wie auch zu mündlichen Erzählformen. Das kommt daher, dass wir eine gewisse Zeit benötigen, um herauszufinden, wie man die digitale Technologie am besten erzählerisch nutzen kann (Klemm 2018: 7). Bis das klar wird, durchläuft jedes neue Medium „eine Phase des Ausprobierens und des Adaptierens bewährter Fähigkeiten an die neuen Rahmenbedingungen [...], ein Learning by doing" (ebd.: 7, Hervorhebung i. O.). Dabei möchten wir einerseits die ,bisherige Praxis möglichst beibehalten“ (ebd., stilistisches Trägheitsprinzip) und andererseits mit den innovativen Möglichkeiten experimentieren. In diesen Prozess gehen jegliche Erzählpraktiken bzw. -traditionen ein (mündlich wie schriftlich), die außerhalb der digitalen Medien liegen. Entsprechend werden auch Ansätze aus der Gesprächsforschung für Erzählungen im Internet interessant. Für die vorliegende Arbeit handelt es sich hierbei um das Konzept der Small Story (Bamberg/Georgakopoulou 2008) und das Erzählen als Kontinuum nach Ochs und Caps (2001). Mit Blick auf das Erzählen in den sozialen Medien (insbesondere auf Twitter und Facebook) beziehen sich die angeführten Forscherinnen und Forscher auf das Konzept der Small Story. Page $(2012,2015)$ und Georgakopoulou (2015) verbinden in ihren Untersuchungen das Konzept der Small Story zusätzlich mit dem Erzählen als Kontinuum. Sie plädieren in Anlehnung an das Erzählmodell von Ochs und Capps (2001: 19 f.) 
dafür, weniger von distinkten Merkmalen, sondern vielmehr von Dimensionen zu sprechen, die für Narrative relevant und unterschiedlich ausgeprägt sind. Da ich die Perspektive ebenfalls für das narrative Vermittlungsvorkommen auf Facebook und Twitter einnehme, gehe ich darauf abschließend ein.

Das Konzept von Small Stories haben Bamberg/Georgakopoulou (2008) maßgeblich vorangetrieben. Sie fassen darunter Erzählungen, die nicht prototypisch ausgearbeitet sind. Solche textuell prototypischen Definitionskriterien beschreiben sie folgendermaßen:

„A coherent temporal progression of events that may be recorded for rhetorical purposes and that is typically located in some past time and place. A plotline that encompasses a beginning, a middle, and an end, conveys a particular perspective and is designed for a particular audience who apprehend and shape its meaning." (Bamberg/ Georgakopoulou 2008: 381)

Konträr zu diesen Merkmalen sollen mit Small Stories Erzählungen erfasst werden, die alltäglich, marginal erscheinen mögen oder die nur angedeutet sind und nicht unbedingt von einem vergangenen abgeschlossenen Ereignis handeln (Bamberg/Georgakopoulou 2008: 377 ff.). Im Sinne rekonstruktiver Gattungen (Bergmann/Luckmann 1995) erfassen und vergegenwärtigen Erzählerinnen in „kleinen“ Erzählungen „Ausschnitte der gesellschaftlichen Wirklichkeit, Erfahrungen und Handlungen in alltäglichen Situationen“ (Günthner 2012: 67). Auch wenn die mündliche Gesprächsforschung ,kleine“ Erzählungen schon früh im Blick hatte, fokussieren Forscher/innen sie gegenwärtig wieder stärker. Ursächlich dafür ist sicher auch das wachsende Interesse am Erzählen in den digitalen Medien, durch das deutlich wird, dass jene ursprünglich prototypischen narrativen Merkmale in der Erzählumgebung nicht immer in Gänze erfüllt sind. Um nicht die Perspektive ,,anything goes“ und ,everything is a small story“ (Georgakopoulou 2015: 256) einzunehmen, schlagen Bamberg und Georgakopoulou einen Mittelweg vor, der es erlaubt, Flexibilität und Relativität in die Definition, was eine Erzählung ist, einzubetten. Zusammen mit Ochs und Capps (2001: 19 f.) sehen sie Erzählungen in verschiedenen Dimensionen realisiert, die in unterschiedlicher Ausprägung vorkommen können. (Bamberg/Georgakopoulou 2008: 382)

Ochs und Caps (2001) konzipieren Erzählen als ein Kontinuum, das von prototypischen bis zu unspezifischen Erzählungen reicht. Letztere fallen häufig aus dem narrativen Untersuchungsrahmen heraus. Erzählungen, die zwischen den zwei Eckpunkten liegen, erfassen die Autorinnen anhand von fünf Grunddimensionen, die wiederum unterschiedlich ausgeprägt sein können. Page (2012, 2015) 
folgt diesem Ansatz und wendet ihn wie folgt für das Erzählen in den sozialen Medien an (Tab. 4.1):

Tab.4.1 Narrative Dimensionen und deren Realisierungsmöglichkeiten in Small Stories (angepasst an Ochs/Capps 2001: 22 ff., nach Page 2015: 333)

\begin{tabular}{l|l}
\hline Grunddimensionen & $\begin{array}{l}\text { Kontinuum: Spektrum der } \\
\text { Realisierungsmöglichkeiten }\end{array}$ \\
\hline $\begin{array}{l}\text { Erzählerschaft: } \\
\text { Wer erzählt? }\end{array}$ & $\begin{array}{l}\text { Eine Erzählung wird von einem/r Erzähler/in } \\
\text { oder von einer multiplen Erzählerschaft } \\
\text { erzählt. }\end{array}$ \\
\hline $\begin{array}{l}\text { Erzählwürdigkeit: } \\
\text { Inwiefern ist die Geschichte } \\
\text { erzählenswert? }\end{array}$ & $\begin{array}{l}\text { Eine Geschichte ist offenkundig wert erzählt } \\
\text { zu werden oder scheinbar irrelevant. }\end{array}$ \\
\hline $\begin{array}{l}\text { Einbettung: } \\
\text { Wie ist die Erzählung in den Kontext }\end{array}$ & $\begin{array}{l}\text { Eine Geschichte ist in ihren Kontext } \\
\text { eingebettet oder von ihm gelöst. }\end{array}$ \\
\hline $\begin{array}{l}\text { Linearität: } \\
\text { In welcher Reihenfolge wird erzählt? }\end{array}$ & $\begin{array}{l}\text { Eine Geschichte ist zeitlich geschlossen, offen } \\
\text { oder multilinear. }\end{array}$ \\
\hline $\begin{array}{l}\text { Moralische Haltung/Wertung: } \\
\text { Wie wird die Erzählung bewertet? }\end{array}$ & $\begin{array}{l}\text { Die Einstellung des Erzählers/der Erzählerin } \\
\text { zu den erzählten Ereignissen ist deutlich oder } \\
\text { verdeckt. }\end{array}$ \\
\hline
\end{tabular}

Die benannten Merkmalsdimensionen sind für die Arbeit aus folgenden Gründen relevant: Ein Merkmal von „kleinen“ im Gegensatz zu „großen“ Erzählungen ist, dass sie in erster Linie kurz sind und sich ,im Geiste einer spätmodernen Konzentration auf die mikrofeinen Aspekte gelebter Erfahrung beziehen“ (Bamberg/ Georgakopoulou 2008: 379, Übersetzung U.A.) können. Ausgehend von dieser Option greifen Unternehmensvertreter/innen in ihren ,kleinen“ Erzählungen gezielt relevante Momente eines Ereignisses heraus. Dadurch können sie bei ihren Rezipient/innen die Vorstellung einer bestimmten „Unternehmenswirklichkeit“ implizieren und/oder affektive Momente betonen.

Des Weiteren ist das Erzählen als Kontinuum aufgrund der Annahme relevant, dass narrative Merkmale in unterschiedlichem Grad vertreten sein können: Das Modell bezieht zwar weiterhin prototypische Definitionskriterien für Erzählungen ein, aber lässt auch narrative Realisierungen $\mathrm{zu}$, die ansonsten aus dem Untersuchungsrahmen herausfallen würden. In der Praxis betrifft das insbesondere Erzählungen über ein oder mehrere Ereignisse, die zumeist jüngst stattfanden oder die noch im Prozess sind. Eine solche Geschichte ist deswegen nicht geschlossen. 
Darüber hinaus bezieht die Frage danach, was als Erzählung gilt, den Anlass, das lokale Verständnis und den Kontext ein. So lässt sich die Erzählberechtigung situativ nicht nur aus dem Ereignis, sondern auch aus dem Veröffentlichungshintergrund ableiten. Das bedeutet: Thematisiert ein Unternehmen im Rahmen seiner externen Kommunikation etwas öffentlich im Internet, ist von einer inhärenten Ereigniswürdigkeit auszugehen, sonst würde es dafür keine Zeit und keine Ressourcen investieren. Aus Sicht der Unternehmen liegt demnach für jeden ihrer narrativen Beiträge eine Erzählberechtigung vor. Umgekehrt stehen sie allerdings auch vor der Aufgabe, diese Erzählberechtigung den Rezipient/innen zu vermitteln.

Open Access Dieses Kapitel wird unter der Creative Commons Namensnennung 4.0 International Lizenz (http://creativecommons.org/licenses/by/4.0/deed.de) veröffentlicht, welche die Nutzung, Vervielfältigung, Bearbeitung, Verbreitung und Wiedergabe in jeglichem Medium und Format erlaubt, sofern Sie den/die ursprünglichen Autor(en) und die Quelle ordnungsgemäß nennen, einen Link zur Creative Commons Lizenz beifügen und angeben, ob Änderungen vorgenommen wurden.

Die in diesem Kapitel enthaltenen Bilder und sonstiges Drittmaterial unterliegen ebenfalls der genannten Creative Commons Lizenz, sofern sich aus der Abbildungslegende nichts anderes ergibt. Sofern das betreffende Material nicht unter der genannten Creative Commons Lizenz steht und die betreffende Handlung nicht nach gesetzlichen Vorschriften erlaubt ist, ist für die oben aufgeführten Weiterverwendungen des Materials die Einwilligung des jeweiligen Rechteinhabers einzuholen.

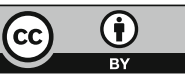

